\title{
Association of liver biomarkers and cytokeratin-18 in Nonalcoholic fatty liver disease patients
}

\author{
Benash Altaf ${ }^{1}$, Anam Rehman², \\ Shireen Jawed ${ }^{3}$, Abdul Raouf ${ }^{4}$
}

\begin{abstract}
Objective: To investigate the association of gold standard liver biomarkers with serum cytokeratin 18 (CK18), serum Alanine aminotransferase (ALT) and serum aspartate (AST).

Methods: This was cross sectional study. It was conducted at Mayo Hospital from January 2016 to December 2017. It comprised of 148 non-alcoholic fatty liver disease subjects of age 40-60 years. After written informed consent, study anthropometric measurements (age, height, waist circumference and hip circumference) were taken and serum AST, ALT and CK-18 were estimated by sandwiched ELISA technique. Data was analyzed using SPSS 21.0. Descriptive were presented as mean and standard deviation. Association between CK18, serum AST and ALT were analyzed by regression analysis and are presented as beta coefficient. P-value $\leq 0.05$ was taken as significant.
\end{abstract}

Results: Study comprised of 148 subjects with mean age $44.81 \pm 6.2$. Of total population $29.1 \%$ were male and $70.9 \%$ were female. Significant positive association of CK18 was found with serum ALT (P-value $0.005^{*}$ ). However, no association was found between AST and serum CK18. (P-value 0.29). Conclusion: Significant positive association was found between Serum CK18 and serum ALT.

KEYWORDS: Cytokeration18, Liver biomarkers, Non-Alcoholic fatty liver disease.

How to cite this:

doi: https://doi.org/10.12669/pjms.36.3.1674

Altaf B, Rehman A, Jawed S, Raouf A. Association of liver biomarkers and cytokeratin-18 in Nonalcoholic fatty liver disease patients. Pak J Med Sci. 2020;36(3):387-390. doi: https://doi.org/10.12669/pjms.36.3.1674

This is an Open Access article distributed under the terms of the Creative Commons Attribution License (http://creativecommons.org/licenses/by/3.0), which permits unrestricted use, distribution, and reproduction in any medium, provided the original work is properly cited.

1. Dr. Benash Altaf, MBBS, M.Phil.

Assistant Professor, Department of Physiology,

2. Dr. Anam Rehman, MBBS, M.Phil.

Senior Demonstrator, Department of Physiology,

3. Dr. Shireen Jawed, MBBS, M. Phil.

Associate Professor, Department of Physiology,

4. Dr. Abdul Raouf,

Assistant Professor, Department of Radiology,

Allied Hospital,

Faisalabad Medical University,

Faisalabad, Pakistan.

1-3: Aziz Fatimah Medical and Dental College,

Faisalabad, Pakistan.

Correspondence:

Dr. Benash Altaf, MBBS, M.Phil.

Assistant Professor,

Department of Physiology,

Aziz Fatimah Medical and Dental College,

Faisalabad, Pakistan.

Email: drshireenjawed@gmail.com

* Received for Publication:

September 18, 2019

* $1^{\text {st }}$ Revision Received:

* $2^{\text {nd }}$ Revision Received:

* Final Revision Accepted:
November 18, 2019

January 27, 2020

February 4, 2020

\section{INTRODUCTION}

Non-alcoholic fatty liver disease (NAFLD) is becoming prevalent due to progressive increase in prevalence of obesity and diabetes. It is involving more than one fourth of the adults globally with $25 \%$ prevalence rate. ${ }^{1}$ NAFLD is one of the utmost common reasons of chronic liver diseases leading to complications like simple steatosis, nonalcoholic steatohepatitis (NASH), fibrosis, and eventually hepatocellular carcinoma (HCC) which is the foremost indication for liver transplantation. ${ }^{2}$ Hence, timely diagnosis and disease progression is important in order to avoid life threatening complications. For diagnosis, ultrasonography, computed tomography (CT) are helpful but liver biopsy is considered as the gold standard.

On histology, NAFLD is depicted by the presence of hepatic steatosis which is excess lipid accumulation without any significant 
inflammation. Whereas NASH is well defined histologically by the presence of hepatic steatosis along with the inflammation of lobules and the hepatocellular degeneration with or without the pericellular fibrosis. ${ }^{3}$

NASH comprises of a spectrum of pathological changes in liver including apoptosis, fibrosis leading to cirrhosis or the hepatocellular carcinoma that involves $20 \%$ of the population. Inspite of NAFLD being epidemic in chronic liver disease, the exact diagnostic options are still needed, so adding up the economic burden on the system of healthcare. ${ }^{2}$

Currently, liver biopsy is the gold standard for NAFLD diagnosis and to assess its progression. However, this procedure has some significant restrictions. First of all, it is invasive requiring hospitalization. Secondly, it might result in crucial life threatening complications like hemorrhage etc. Furthermore, ethically it is not possible to perform biopsy repeatedly in NAFLD subjects to see the disease progression. The relative complications and high expenses of gold standard test utilized for the diagnosis of NAFLD has insisted the field to pursuit for more diagnostic methods. ${ }^{4}$ Evidence based researches have suggested that serum biomarkers including serum AST, ALT and CK18 have a remarkable role in early detection of the liver diseases. ${ }^{5}$ Elevation of serum transaminase levels in NAFLD patients has been well documented by previous studies. On the other hand, serum ALT is generally higher than AST in diseased liver conditions. However, some recent studies were not in agreement with these findings. Many patients with advance fibrosis and NASH can also present with normal liver enzymes. ${ }^{6}$

CK18 is present in huge amount in liver. It is an intermediate filament protein representing $5 \%$ of the hepatic proteins. In the apoptosis of cell, CK18 is cleaved and the fragments of CK18 are identified by M30 antibody specific for liberated C-terminus. Aim of the study was to find the association of liver biomarkers serum AST and ALT with CK18 which can be used as diagnostic biomarker as well as marker for disease progression in future.

\section{METHODS}

This was a cross sectional study, conducted at outpatient Department of Radiology of Mayo Hospital, King Edward Medical University, Lahore, after taking ethical approval (134/RC/
KEMU) from January 2016 to December 2017. Total 148 NAFLD subjects were recruited by non-probability convenient sampling technique. NAFLD subjects with fatty liver were diagnosed on ultrasonography with high echogenic shadows. Known diabetic subjects, subjects suffering from any type of hepatitis, taking homeopathic medications or glucocorticoids and taking alcohol were excluded from the study. Nondiabetic subjects of both gender of age ranging 40-60 years with fatty liver were included in this study. Pre-screening was done for fasting blood sugar levels with the help of glucometer (model U-RIGHTTD -4251) to exclude diabetic subjects. Only non-diabetic subjects with blood sugar levels less than $126 \mathrm{~g} / \mathrm{dl}$ were included in this study. Anthropometric measures including height, waist circumference and hip circumference were measured without shoes following the protocol. Under aseptic measures, 5cc of blood samples were drawn for measuring the serum AST, ALT and CK18 levels. After centrifuging the blood at $3000 \mathrm{rev} / \mathrm{min}$ for $15-20$ minutes, serum was stored in ependendroff tubes at $-70^{\circ} \mathrm{C}$ in pathology laboratory of King Edward Medical University till samples were run on ELISA.

Statistical Analysis: The data was analyzed using SPSS 21.0. Descriptive were presented as mean \pm SD. Normality of data was checked by Shapiro's wilk test with value $\leq 0.05$, showing data is not normally distributed. Regression analysis was applied to explore the association of CK18 with standard liver markers AST and ALT. P-value $\leq$ 0.05 was considered significant.

\section{RESULTS}

This study comprised of 148 subjects with mean age $44.81 \pm 6.2$. Out of this $105(70.9 \%)$ were female subjects and $43(29.1 \%)$ were male. The anthropometric and biochemical descriptive of the studied population are shown in Table-I. Significant positive association of CK18 with serum ALT was found (P-value $0.05^{*}$ ), but serum AST was not significantly associated with CK18 (P-value 0.29) (Table-II).

\section{DISCUSSION}

NAFLD is a global health issue and its accurate diagnosis is made on liver biopsy. As of care and financial perspectives, liver biopsy is not possible to be done in each patient suffering from NAFLD. ${ }^{7}$ Due to significant rise in the prevalence of NAFLD 
Table-I: Descriptive Statistics of Studied Subjects $(\mathrm{n}=148)$.

\begin{tabular}{lcc}
\hline $\begin{array}{l}\text { Anthropometric and } \\
\text { Biochemical Statistics }\end{array}$ & Mean $\pm S D$ & $\begin{array}{c}\text { Median } \\
(I Q R)\end{array}$ \\
\hline Age (years) & $44.81 \pm 6.20$ & $43(48-40)$ \\
Height (m) & $1.59 \pm 0.06$ & $1.6(1.65-1.57)$ \\
Waist (cm) & $38.67 \pm 3.66$ & $38(40-38)$ \\
Hip (cm) & $41.38 \pm 3.95$ & $41(42.0-40.0)$ \\
(ALT) (35U/L) & $20.04 \pm 20.92$ & $15.0(21.7-11.0)$ \\
(AST) (30U/L) & $20.99 \pm 25.02$ & $15.0(23.7-9.2)$ \\
(CK-18) (U/L) & $13.95 \pm 19.84$ & $9.3(14.1-5.1)$ \\
\hline
\end{tabular}

ALT: Alanine Transaminase, AST: Aspartate Transaminase,

CK18: Cytokeratin-18, IQR: Interquartile range, SD: Standard Deviation, m: meter, $\mathrm{cm}$ : centimeter, U/L: units per liter.

in concurrence with the remarkable research works for making novel therapies for patients, non-invasive, appropriate and dependable serum biomarkers are really required. ${ }^{4}$ It is noticeable that the laboratory tests done in routine for the assessment of subjects assumed to be NAFLD comprises of a long list of liver biomarkers like serum ALT, AST, alkaline phosphatase(ALP), and gamma-glutamyl-transpeptidase. Various studies have documented that the whole histological picture of NAFLD can be appreciated in patients with normal levels of ALT. ${ }^{6}$ Raised levels of serum ALT and AST might be present in NAFLD patients, though levels within normal do not eliminate the existence of NAFLD and NASH. ${ }^{7}$ When raised, these patients mostly have serum AST to ALT ratios of less than 1, distinct to the alcoholic liver disease in which specifically the ratios are greater indicating that there is no alteration in the degree of advanced fibrosis among normal and the group with raised ALT levels. Furthermore, it is also shown that serum ALT levels two times more than the maximum limit of normal have restricted efficacy in expecting NAFLD with a specificity of $61 \%$ and sensitivity of $50 \% .^{1}$ (This is the reason that various researches are exploring the diagnostic biomarkers that can be helpful in making the diagnosis. The recent American guidelines provided for making the diagnosis and management of the NAFLD states that serum CK18 levels are useful for diagnostic purpose as well as it also subsidizes in the disease progression. But it is suggested that the usage of CK-18 in routine clinical practice is premature. ${ }^{8}$ Current studies have documented CK18 as convenient biomarker
Table-II: Association of CK18 with Liver Biomarkers.

\begin{tabular}{lccc}
\hline $\begin{array}{l}\text { Independent } \\
\text { Variables }\end{array}$ & $\begin{array}{c}\text { Beta Coefficient } \\
(\beta)\end{array}$ & P-value & $\begin{array}{c}\text { Confidence } \\
\text { Interval }\end{array}$ \\
\hline AST & 0.051 & 0.29 & $-0.117-0.219$ \\
ALT & 1.62 & $0.05^{*}$ & $-0.153-0.249$ \\
\hline
\end{tabular}

Dependent Variable: CK18.

for making the diagnosis of the NAFLD. Musso $\mathrm{G}$ also reported similar results concerning CK18 and described it as a biomarker of hepatocyte apoptosis that can be used to evaluate the disease progression. $^{9}$

Moreover, some studies have stated that the accuracy of serum ALT is relatively poor just at $40 \%$ for making the diagnosis of NAFLD. ${ }^{6}$ Due to the restrictions of serum transaminases as non-invasive biomarkers of NAFLD, irresistible evidences exhibited that definite cytokines which are derived of various biochemical events comprising of resistance to insulin, oxygen stress, apoptosis and inflammation play significant role in NAFLD progression. Similarly, throughout the hepatocyte apoptosis, the cleaved pieces of CK18 are detectable in patient serum suffering from chronic liver disease. This technique was verified as a capable non-invasive tool in NAFLD/NASH diagnosis. ${ }^{6}$ Swiderska M and his colleges found altered levels of CK18 depending upon the severity of disease inspite of having normal serum ALT. ${ }^{10}$ Incontrast to this, our study found positive association of CK18 with serum ALT. Study conducted by Alt $\mathrm{Y}$ and his colleagues are in agreement to our results concerning serum ALT as they reported similar association. However, as regards serum AST they reported contradictory findings to our result as they found significant positive association of serum AST with CK18. ${ }^{11}$

NAFLD exerts considerable negative impact on the patient's health-related quality. By estimating progression of disease earlier precautionary measures could be adopted to prevent further progression of NAFLD. Noninvasive biomarkers should be applied to clinical practice to reduce unnecessary biopsies. ${ }^{12}$ In future, CK18 can be used for monitoring the disease status as well as evaluating the response to the treatment in NAFLD. Similar researches concerning CK18 and other diagnostic biomarkers should be conducted on a broader scale. ${ }^{13}$ 


\section{CONCLUSION}

CK18 is positively associated with ALT. Future researches on broader scales are required to validate this association.

Conflict of interest: None.

Funding support: KEMU.

\section{REFERENCES}

1. Araujo AR, Rosso N, Bedogni G, Tiribelli C, Bellentani S. Global epidemiology of non-alcoholic fatty liver disease/ non-alcoholic steatohepatitis: What we need in the future Liver Int. 2018;38(S1):47-51. doi: 10.1111/liv.13643

2. Parkash $\mathrm{O}$, Hamid S. We ready for a new epidemic of under recognized liver disease in South Asia especially in Pakistan? Non alcoholic fatty liver disease. J Pak Med Assoc. 2013;63(1):95-99.

3. Younossi ZM, Blissett D, Blissett R, Henry L, Stepanova $\mathrm{M}$, Younossi $\mathrm{Y}$ et al. The economic and clinical burden of nonalcoholic fatty liver disease in the United States and Europe. Hepatology. 2016;64(5):1577-1586. doi: 10.1002/ hep. 28785

4. He L, Deng L, Zhang Q, Guo J, Zhou J, Song W, et al. Diagnostic value of CK-18, FGF-21, and related biomarker panel in nonalcoholic fatty liver disease: a systematic review and meta-analysis. Biomed Res Int. 2017;2017:9729107. doi: $10.1155 / 2017 / 9729107$

5. Ratziu V, Bellentani S, Cortez-Pinto H, Day C, Marchesini G. A position statement on NAFLD/NASH based on the EASL 2009 special conference. J Hepatol. 2010;53:372-384.

6. Pepe MS, Etzioni R, Feng Z, Potter JD, Thompson ML, Thornquist $M$, et al. Phases of biomarker development for early detection of cancer. J Natl Cancer Inst. 2001;93:1054-1061.

7. Taseer IH, Hussain L, Safdar S, Mirbahar AM, Ahmad I. Frequency of non alcoholic fatty liver disease (NAFLD) \& its biochemical derangements in Type-2 diabetic patients. Pak J Med Sci. 2009;25(5):817-820.

8. Kawanaka M, Nishino K, Nakamura J, Urata N, Oka T, Goto $\mathrm{D}$, et al. Correlation between serum cytokeratin-18 and the progression or regression of non-alcoholic fatty liver disease. Ann Hepatol. 2015;14(6):837-844. doi: 10.5604/16652681
9. Musso G, Gambino R, Cassader M, Pagano G. Metaanalysis: natural history of non-alcoholic fatty liver disease (NAFLD) and diagnostic accuracy of non-invasive tests for liver disease severity. Ann Med. 2011;43:617-649.

10. Swiderska M, Jaroszewicz J, Parfieniuk-Kowerda A, Rogalska-Plonska M, Stawicka A, Panasiuk A, et al. Serum Cytokeratin 18 M30 Levels in Chronic Hepatitis B Reflect Both Phase and Histological Activities of Disease. Mediators Inflamm. 2017;2017:3480234. doi: 10.1155/2017/3480234

11. Alt $\mathrm{Y}$, Grimm A, Schlegel L, Grambihler A, Jens $M$, Wiltink KJ, et al. The Impact of Liver Cell Injury on Health-Related Quality of Life in Patients with Chronic Liver Disease. PLoS One. 2016;11(3):e0151200. doi: 10.1371/journal.pone.0151200

12. Ji $Y X$, Huang $Z$, Yang $X$, Wang $X$, Zhao LP, Wang PX, et al. The deubiquitinating enzyme cylindromatosis mitigates nonalcoholic steatohepatitis. Nature Med. 2018;24(2):213223. doi: $10.1038 / \mathrm{nm} .4461$

13. Tsutsumi $M$, Tanaka N, Kawakubo $M$, Sheena $Y$, Horiuchi A, Komatsu M, et al. Serum fragmented cytokeratin 18 levels reflect the histologic activity score of nonalcoholic fatty liver disease more accurately than serum alanine aminotransferase levels. J Clin Gastroenterol. 2010;44(6):440-447.

\section{Author Contribution:}

Benash Altaf helped in Study design, data collection, Interpretation of results, manuscript writing and is accountable for the integrity of the data.

Anam Rehman helped in manuscript writing and drafting, reviewed and approved the manuscript.

Shireen Jawed helped in statistical analysis, interpretation of results, formulation of tables, writing the manuscript. Reviewed and approved the manuscript

Abdul Raouf helped in manuscript writing and critically reviewed it for intellectually content. 